\title{
Naphthalocyanine Thin Films and Field Effect Transistors
}

\author{
Ayad M.S. Esmail, Chrisopher R. Staddon and Peter H. Beton* \\ School of Physics \& Astronomy, University of Nottingham, Nottingham NG7 2RD, UK \\ *corresponding author peter.beton@nottingham.ac.uk
}

\section{ABSTRACT}

Naphthalocyanine $(\mathrm{Nc})$ thin films have been grown by sublimation on $\mathrm{SiO}_{2}$. We have used atomic force microscopy and X-ray diffraction to show that the films are disordered for room temperature deposition, but show a highly crystalline needle-like morphology for a substrate temperature of $\sim 200^{\circ} \mathrm{C}$. Field effect transistors exhibit $p$-channel operation with a mobility, which has a peak value of $0.052 \mathrm{~cm}^{2} / \mathrm{Vs}$, showing a high dependence on substrate temperature. Exposure to atmosphere results in an increase in current and mobility and a reduction in threshold voltage. We compare our results with films formed from analogue molecules such as phthalocyanines and naphthalocyanine functionalised with solubilising sidegroups and discuss the potential of Nc for applications in organic electronics and sensors. 


\section{INTRODUCTION}

Dye molecules derivatised from phthalocyanine (Pc) and the larger analogue molecule, naphthalocyanine (Nc) have attracted much attention as candidate materials for photovoltaic devices, field effect transistors, gas sensing and organic spintronic devices ${ }^{1-13}$. The parent molecules are planar with a central conjugated ring system which can accommodate a metal centre through the formation of co-ordination bonds. In the solid state the molecules stack face-to-face which leads to the formation of needle-like crystals. Both molecules strongly adsorb light and the larger molecule, Nc, has a more extended $\pi$ conjugation system, resulting in an adsorption edge at longer wave length, in the infra-red. Both molecules are highly insoluble and there have been many studies of derivatives which have additional groups which can promote solubility ${ }^{4,13}$. Pc can be sublimed readily and the resulting thin films have been incorporated into a wide range of organic devices ${ }^{1-3,5}$. However, there have so far been very few studies of the properties of sublimed Nc thin films $^{11,14,15}$; in fact it has been suggested that Nc cannot be sublimed ${ }^{13}$, although the deposition of monolayer coverages have been reported by a number of groups ${ }^{16-22}$. Consequently it has not been possible to characterise systematically the properties of sublimed Nc thin films.

In this paper we investigate the growth of Nc thin films by sublimation and show that the resulting organic layers can be used to form p-type field effect transistors which can be operated under both vacuum and atmospheric conditions. We use atomic force microscopy (AFM) and X-ray diffraction (XRD) to investigate the grown films and find a strong temperature dependence of morphology with the formation of highly-facetted needle-like crystals for elevated substrate temperatures during growth. 


\section{EXPERIMENTAL}

For substrates we use pieces (typically $1 \times 1 \mathrm{~cm}^{2}$ ) of a Si wafer on which a $200 \mathrm{~nm}$ thermal oxide has been grown. For bottom-contact devices the substrates are pre-patterned with a contact metallisation by evaporating $10 \mathrm{~nm}$ Ti followed by $100 \mathrm{~nm}$ of Au through a shadow mask to define source and drain contacts with typical length $L=25 \mu \mathrm{m}$, and width $w=3$ $\mathrm{mm}$. To sublime Nc these samples are then transferred via atmosphere to a separate vacuum system where they are mounted on a heater in an arrangement which permits insitu electrical measurements through the use of mechanical probes. Top-contact devices are fabricated by first depositing the Nc layer, then transferring the sample via atmosphere to a vacuum system for metallisation using the same shadow mask arrangement as described above. Nc (purchased commercially from Sigma; structure shown in Figure 1a) is loaded into a quartz crucible and annealed for approximately 12 hours prior to sublimation at a rate, measured using a quartz crystal thickness monitor, of $20 \mathrm{~nm} /$ hour. The base pressure of the vacuum system was $2 \times 10^{-7} \mathrm{mbar}$ and the substrate temperature, $T_{s}$, is measured using a thermocouple.

Electrical measurements were acquired in the vacuum system in the dark either at the base pressure, or at atmospheric pressure after the introduction of air to the chamber in which the sample is housed. Top-contact devices were pre-annealed at $100^{\circ} \mathrm{C}$ for 2 hours prior to the acquisition of electrical characteristics. 
The Nc films were characterised structurally using an Asylum MFP3D atomic force microscope (AFM) operated in AC (tapping) mode and the orientational order relative to the substrate was determined using $\theta-2 \theta$ X-ray diffraction scans.

\section{RESULTS AND DISCUSSION}

Figure 1b-d shows AFM images of samples after the deposition of $65 \mathrm{~nm}$ of Nc at substrate temperatures, $\mathrm{T}_{\mathrm{s}}$, varying from room temperature up to $230^{\circ} \mathrm{C}$. For low temperatures we observe islands with typical lateral dimensions of $40 \mathrm{~nm}$ and a near-circular shape with no obvious faceting. As the substrate temperature is progressively increased the islands evolve into a more asymmetric facetted shape, very similar to the needle-like crystals reported ${ }^{1}$ for Pc. In addition, the coverage of the substrate starts to reduce and the sticking coefficient falls to zero for $T_{s}>250^{\circ} \mathrm{C}$.

X-ray diffraction data ( $\theta-2 \theta$ plots) confirm an increase in order with well-defined peaks appearing for films grown at $T_{s}=180^{\circ} \mathrm{C}$ (see Figure 2). The position of the principal peak at $2 \theta=5.84 \pm 0.09^{\circ}$ corresponds to a plane spacing perpendicular to the surface of $1.51 \pm 0.02$ $\mathrm{nm}$. Additional higher order peaks are also observed for this sample, consistent with this plane spacing. At the lower growth temperature of $\mathrm{T}_{\mathrm{s}}=120^{\circ} \mathrm{C}$ the same first order peak is observed by the higher order peaks are much less intense. The sample grown at room temperature is much less ordered with a very broad feature at $2 \theta \sim 6^{\circ}$. Although the crystal structure for metal-free naphthalocyanine has not been published, the observed spacing observed for the samples grown at higher temperature is very close to that observed in the growth of metallated $(\mathrm{Zn})$ naphthalocyanine ${ }^{14}$ and cannot be explained by molecules lying 
parallel to the substrate, for which a spacing of $\sim 0.35 \mathrm{~nm}$ would be expected. Instead we infer that the growth morphology is very similar to that observed for $\mathrm{Pc}^{1}$ in which there is a switch from a flat-lying disordered morphology at low temperatures to a face-to-face packing of molecules aligned out-of-plane with the substrate at elevated temperatures.

The characteristics of Nc field effect transistors (FET) can be measured using the pre-formed contact metallisation as source and drain, and the silicon substrate as the gate in a conventional (bottom-contact) device geometry ${ }^{23}$. The characteristic for a device prepared with a sample temperature of $200^{\circ} \mathrm{C}$ measured in vacuum (see Figure 3a) shows excellent transistor action through the formation of a p-type accumulation channel at a threshold voltage of $\mathrm{V}_{\mathrm{T}}=-20 \mathrm{~V}$ (determined from the corresponding transfer characteristic in Fig. 3b), minimal leakage current and an on-off ratio of $>10^{5}$ (measured at source drain voltage of $60 \mathrm{~V})$. The characteristic in Fig. 3a includes data taken as the source-drain voltage is swept up and down; these plots are overlaid indicating that the measured characteristics are largely free of hysteresis. From Fig. 3b we determine a mobility of $\mu=0.014 \mathrm{~cm}^{2} / \mathrm{Vs}$ (here we use the $\mu=\left(L / w C_{i}\right)\left(\mathrm{dl}^{1 / 2} / \mathrm{dV}_{\mathrm{G}}\right)^{2}$, where $C_{\mathrm{i}}=17 \mathrm{nF} / \mathrm{cm}^{2}$ is the gate capacitance per area, $\mathrm{I}$ is the current and $\mathrm{V}_{\mathrm{G}}$ is the gate voltage.

The exposure to atmosphere has a highly detrimental effect on the electrical characteristics of many organic thin film transistors, but for the Nc transistors we observe an increase in current under these conditions. In Figs. $3 c$ and $3 d$ we show the I(V) characteristics of the device discussed above after the pressure in the chamber has been allowed to rise to atmospheric pressure through the introduction of air. We note that the saturation current has increased by approximately $50 \%$, over the full range of gate voltages (for example at a 
source-drain voltage of -70V. Analysis of the corresponding transfer characteristic in Fig. 3d shows that this rise can be attributed to a increase in mobility to $0.02 \mathrm{~cm}^{2} / \mathrm{Vs}$; we also observe a decrease of the threshold voltage to $\mathrm{V}_{\mathrm{T}}=-13 \mathrm{~V}$. The changes arising from atmospheric exposure may be reversed by annealing to $\sim 100^{\circ} \mathrm{C}$ in vacuum.

The mobility depends strongly on the substrate temperature during growth, as might be expected for the very different morphologies and order determined from AFM and XRD. This variation is summarised in Figure 4 which shows the mobility, both in vacuum and air, for substrate temperatures in the range of $120-250^{\circ} \mathrm{C}$. The maximum mobility occurs at a temperature where the AFM (Fig. 1c) shows a high degree of order, but without the significant gaps and loss of connectivity observed at higher temperature (Fig. 1d). We attribute the fall in the apparent mobility at the very highest temperatures reported in Fig. 4 to the reduction in material adhering to the surface (Fig. 1d); this is not an intrinsic material effect but a consequence of the dramatic reduction of conducting pathways for samples grown with a fixed incident dosage, but lower sticking coefficient.

The increase in mobility on exposure to atmosphere occurs consistently over this temperature range. This is observed more clearly in a plot on a logarithmic scale included in the Supporting Information (SI). This data in $\mathrm{SI}$ also includes measurements of devices grown at room temperature for which the mobility in vacuum (air) is $\sim 10^{-5} \mathrm{~cm}^{2} / \mathrm{Vs}\left(\sim 10^{-4}\right.$ $\mathrm{cm}^{2} / \mathrm{Vs}$ ), several orders of magnitude lower than the maximum value. It is known that an increase in current flowing FETs formed from solubilised Nc derivatives can occur in the presence of oxidising species through their action as electron acceptors ${ }^{9}$. Our observation of a shift of threshold voltage to less negative values is consistent with an increase in hole 
concentration which compensates traps, either in the dielectric layer or at the interfaces of the Nc film. In our case the presence of molecular oxygen due to atmospheric exposure is a likely candidate for the additional acceptors ${ }^{1}$. The increase in mobility can also be explained by the presence of additional acceptors through the compensation and screening of charged traps leading to a reduction in scattering.

We have also fabricated devices in a top contact geometry in which the Nc layer is deposited prior to the deposition of contacts as described above; these devices are passed through the atmosphere then annealed in vacuum prior to the acquisition of electrical measurements such as those shown in Figure 5 (sample grown at $200^{\circ} \mathrm{C}$ ). Similar to the bottom-contact devices we observe transistor action with minimal hysteresis, but the measured mobility is higher, $0.052 \mathrm{~cm}^{2} / \mathrm{Vs}$, and the threshold voltage $\mathrm{V}_{\mathrm{T}}=4 \mathrm{~V}$ so that the device is normally on. This value of mobility is close to the maximum reported for solution processed Nc $\mathrm{FETs}^{9}$. The effect of substrate temperature on the electrical characteristics shows the same general trends as for the bottom contact devices with an increase as the substrate temperature is raised to $\sim 200^{\circ} \mathrm{C}$ followed by a rapid fall as the sticking coefficient is reduced at higher values (these data are included in Fig. 4). However, in the range 120$200^{\circ} \mathrm{C}$ the mobility varies over a smaller magnitude as compared with bottom-contact devices (see also logarithmic plot in SI). The differences between top- and bottom-contact FETs are likely due to changes in the nucleation and growth of Nc close to the gold contacts which are present prior to Nc sublimation for bottom-contact geometry. 


\section{CONCLUSIONS}

Our results confirm that, contrary to some suggestions in the literature ${ }^{13}$, it is possible to fabricate thin film FETs from sublimed Nc layers. The transistor characteristics, and the variation of morphology and carrier mobility with substrate temperature are highly reminiscent of the trends observed for the smaller analogue molecule phthalocyanine and our results suggest that it may be possible to substitute Nc for Pc in applications, such as photovoltaics and photoconductors, where there may be advantages in using sublimed films which exploit the properties of Nc which has an optical absorption edge at a much longer wave length. To this end we plan to investigate the optical properties of sublimed Nc codeposited with other organic molecules in our future work.

\section{ACKNOWLEDGEMENTS}

A.M.S.E. is grateful to the Human Capacity Development Program (HCDP) of the Kurdistan Regional Government for the financial support of his Ph.D. scholarship.

SUPPORTING INFORMATION Additional data on the temperature dependence of the mobility is available online. 


\section{REFERENCES}

(1) Bao, Z.; Lovinger, A.; Dodabalapur, A. Organic Field-effect Transistors with High Mobility Based on Copper Phthalocyanine. Appl. Phys. Lett. 1996, 69, 3066-3068.

(2) Liu, S.; Wang, W. M.; Briseno, A. L.; Mannsfeld, S. C. B.; Bao, Z. Controlled Deposition of Crystalline Organic Semiconductors for Field-Effect-Transistor Applications. Adv. Mater. 2009, 21, 1217-1232.

(3) Lin, Y.; Li, Y.; Zhan, X. Small Molecule Semiconductors for High-Efficiency Organic Photovoltaics. Chem. Soc. Rev. 2012, 41, 4245-4272.

(4) de la Torre, G.; Claessens, C. G.; Torres, T. Phthalocyanines: Old Dyes, New Materials. Putting Color in Nanotechnology. Chem. Commun. 2007, 2000-2015.

(5) Guillaud, G.; Simon, J.; Germain, J. P. Metallophthalocyanines Gas Sensors, Resistors and Field Effect Transistors. Coord. Chem. Rev. 1998, 178-180, 1433-1484.

(6) Bohrer, F. I.; Colesniuc, C. N.; Park, J.; Ruidiaz, M. E.; Schuller, I. K.; Kummel, A. C.; Trogler, W. C. Comparative Gas Sensing in Cobalt, Nickel, Copper, Zinc, and MetalFree Phthalocyanine Chemiresistors. J. Am. Chem. Soc. 2009, 131, 478-485.

(7) Urdampilleta, M.; Klyatskaya, S.; Cleuziou, J.-P.; Ruben, M.; Wernsdorfer, W. Supramolecular Spin Valves. Nat. Mater. 2011, 10, 502-506.

(8) Heutz, S.; Mitra, C.; Wu, W.; Fisher, A. J.; Kerridge, A.; Stoneham, M.; Harker, T. H.; Gardener, J.; Tseng, H. H.; Jones, T. S.; et al. Molecular Thin Films: A New Type of Magnetic Switch. Adv. Mater. 2007, 19, 3618-3622.

(9) Royer, J. E.; Zhang, C.; Kummel, A. C.; Trogler, W. C. Air-Stable Spin-Coated Naphthalocyanine Transistors for Enhanced Chemical Vapor Detection. Langmuir 2012, 28, 6192-6200.

(10) Royer, J. E.; Kappe, E. D.; Zhang, C.; Martin, D. T.; Trogler, W. C.; Kummel, A. C. 
Organic Thin-Film Transistors for Selective Hydrogen Peroxide and Organic Peroxide Vapor Detection. J. Phys. Chem. C 2012, 116, 24566-24572.

(11) Farag, A. A. M.; Yahia, I. S.; AlFaify, S.; Al-Ghamdi, A. A. Nano-Flower 2,3Naphthalocyanine Heterojunction for Optoelectronic Applications. Synth. Met. 2015, 203, 261-268.

(12) Pandey, R.; Kerner, R. A.; Menke, S. M.; Holst, J.; Josyula, K. V. B.; Holmes, R. J. Tin Naphthalocyanine Complexes for Infrared Absorption in Organic Photovoltaic Cells. Org. Electron. 2013, 14, 804-808.

(13) Kikukawa, Y.; Fukuda, T.; Fuyuhiro, A.; Ishikawa, N.; Kobayashi, N. Facile One-Pot Preparation of Thermally and Photochemically Convertible Soluble Precursors of Copper Phthalocyanine and Naphthalocyanine. Chem. Commun. 2011, 47, 85188520.

(14) Yanagi, H.; Kouzeki, T.; Ashida, M.; Noguchi, T.; Manivannan, A.; Hashimoto, K.; Fujishima, A. Molecular Orientation of Vacuum-Deposited Thin Films of Zincnaphthalocyanine. J. Appl. Phys. 1992, 71, 5146-5153.

(15) Yanagi, H.; Kanbayashi, Y.; Schlettwein, D.; Wohrle, D.; Armstrong, N. R. Photoelectrochemical Investigations on Naphthalocyanine Derivatives in Thin-Films. J. Phys. Chem. 1994, 98, 4760-4766.

(16) Wiggins, B.; Hipps, K. W. Investigation of Metal Free Naphthalocyanine Vapor Deposited on Au(111). J. Phys. Chem. C 2014, 118, 4222-4230.

(17) Pham, T. A.; Song, F.; Stöhr, M. Supramolecular Self-Assembly of Metal-Free Naphthalocyanine on Au(111). Phys. Chem. Chem. Phys. 2014, 16, 8881-8885.

(18) Wu, R.; Yan, L.; Zhang, Y.; Ren, J.; Bao, D.; Zhang, H.; Wang, Y.; Du, S.; Huan, Q.; Gao, H. J. Self-Assembled Patterns and Young's Modulus of Single-Layer Naphthalocyanine 
Molecules on $\mathrm{Ag}(111)$. J. Phys. Chem. C 2015, 119, 8208-8212.

(19) Mehring, P.; Beimborn, A.; Lühr, T.; Westphal, C. Metal-Free Naphthalocyanine Structures on $\mathrm{Au}(100)$ at Submonolayer Coverage. J. Phys. Chem. C 2012, 116, 1281912823.

(20) Liljeroth, P.; Repp, J.; Meyer, G. Current-Induced Hydrogen Tautomerization and Conductance Switching of Naphthalocyanine Molecules. Science 2007, 317, 12031206.

(21) Yan, L.-H.; Wu, R.-T.; Bao, D.-L.; Ren, J.-H.; Zhang, Y.-F.; Zhang, H.-G.; Huang, L.; Wang, Y.-L.; Du, S.-X.; Huan, Q.; et al. Adsorption Behavior of Fe Atoms on a Naphthalocyanine Monolayer on $\mathrm{Ag}(111)$ Surface. Chinese Phys. B 2015, 24, 076802/1-076802/5

(22) Berner, S.; Corso, M.; Widmer, R.; Groening, O.; Laskowski, R.; Blaha, P.; Schwarz, K.; Goriachko, A.; Over, H.; Gsell, S.; et al. Boron Nitride Nanomesh: Functionality from a Corrugated Monolayer. Angew. Chem. Int. Ed. Engl. 2007, 46, 5115-5119.

(23) Haddon, R. C.; Perel, A. S.; Morris, R. C.; Palstra, T. T. M.; Hebard, A. F.; Fleming, R. M. C60 Thin Film Transistors. Appl. Phys. Lett. 1995, 67, 121-123. 


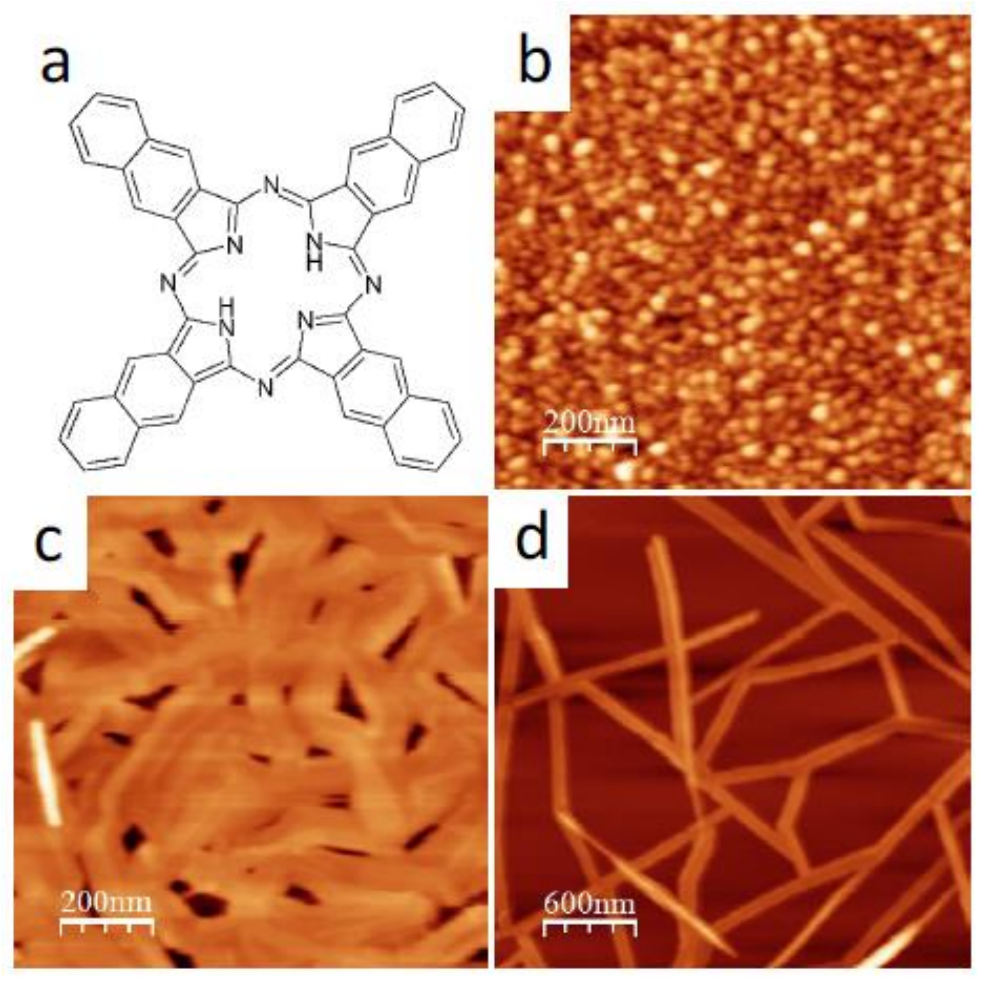

Figure 1 a) schematic of naphthalocyanine; b) - d) AFM images of NC films grown at substrate temperatures, b) room temperature, c) $200^{\circ} \mathrm{C}$, d) $230^{\circ} \mathrm{C}$. At low temperature small grains are observed but at high temperatures needle-like crystallites are formed. 


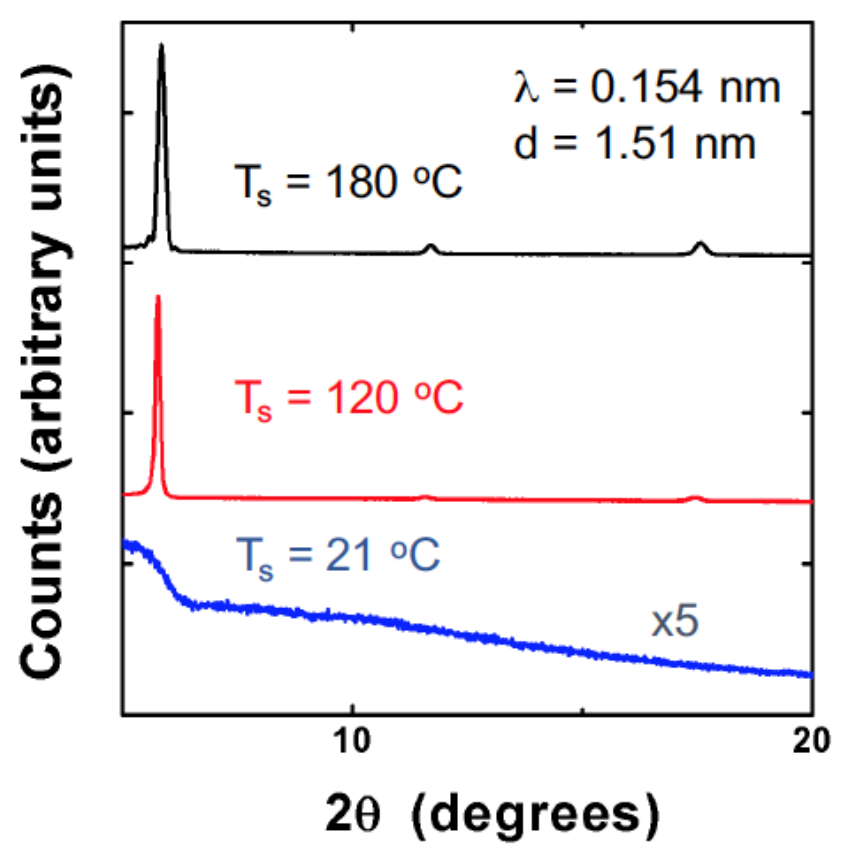

Figure $2 \theta-2 \theta$ X-ray diffraction of Nc films grown at room temperature, $120^{\circ} \mathrm{C}$ and $180^{\circ} \mathrm{C}$.

Films grown at higher temperature show clear peaks indicating the presence of crystalline material with a plane spacing perpendicular to the substrate of $d=1.51 \mathrm{~nm}$. 

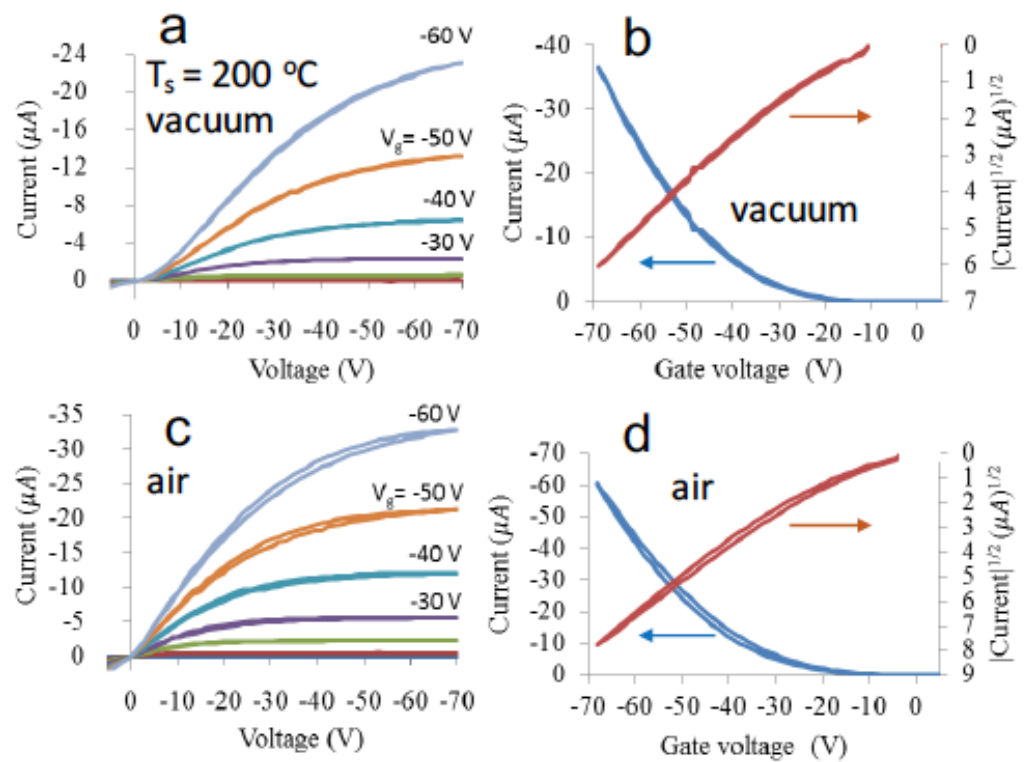

Figure 3 I(V) characteristics of a bottom contact Nc FET grown at $200^{\circ} \mathrm{C}$. a) and b) show, respectively, transistor and transfer characteristics of a device held under vacuum conditions; c) and d) show the transistor and transfer characteristics of the same device after exposure to atmosphere. In all measurements the voltage is ramped up and down. The curves are essentially indistinguishable in a) and b); in c) and d) there is a small amount of hysteresis. 


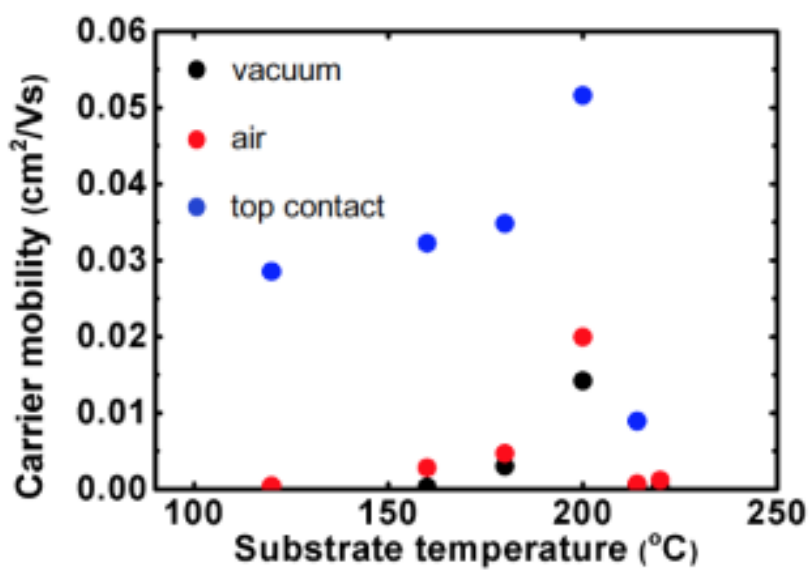

Figure 4 Variation of carrier mobility with substrate temperature for bottom contact devices in vacuum and air and also for top contact devices. 

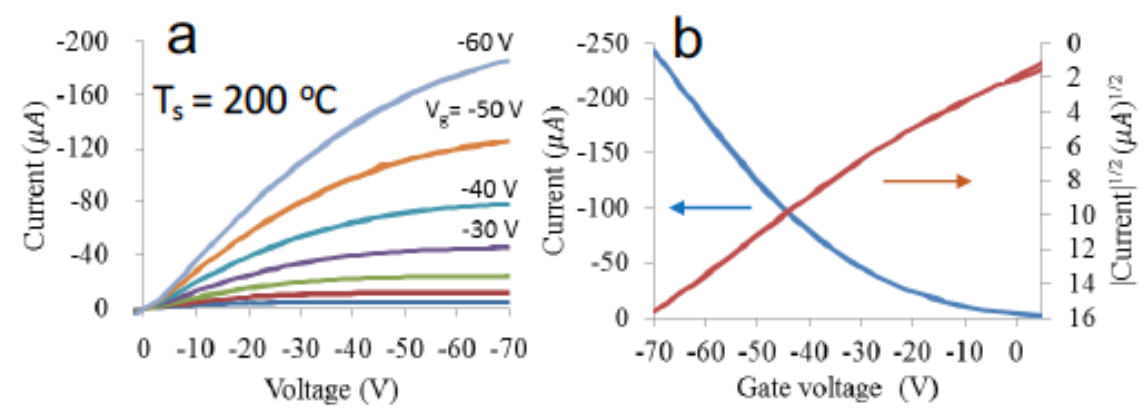

Figure 5 Measured transistor (a) and transfer (b) characteristic of a device grown at $200^{\circ} \mathrm{C}$ and fabricated in a top contact geometry. Measurements were performed at room temperature under vacuum; the sample was first annealed in vacuum at $100^{\circ} \mathrm{C}$. 

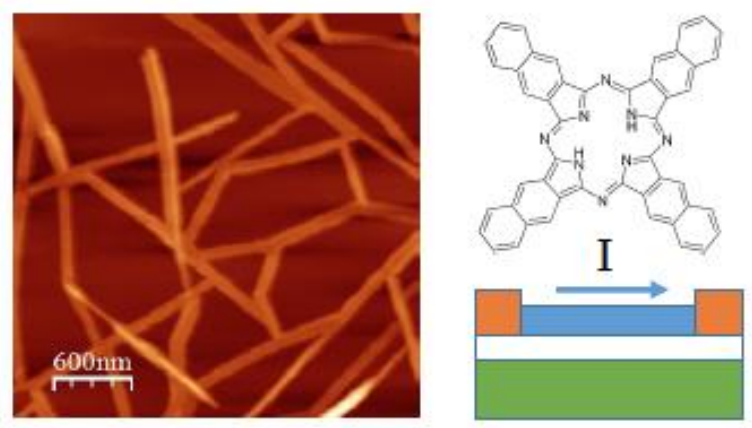

Table of Contents Figure 